\title{
Abordaje quirúrgico de los dientes supernumerarios
}

CAsos Clínicos

Surgical approach about supernumerary teeth.

\section{Resumen}

Este reporte de caso clínico presenta la secuencia clínica del tratamiento quirúrgico de un diente supernumerario en posición transversal. El tratamiento quirúrgico (exodoncia) de los dientes supernumerarios están indicados en piezas incluidas y que están además dentro de las arcadas dentarias; esta patología odontogénica suele ser encontrada de manera esporádica durante los exámenes complementarios; sin embargo, el no abordarlo y tratarlo pueden devenir en otra patología dentaria que involucre piezas adyacentes a la lesión. Se presenta la secuencia clínica del abordaje quirúrgico para extraer una pieza dentaria supernumeraria en posición transversal.

Palabras claves: Diente supernumerario, exodoncia, odontopediatría.

\section{Abstract}

This clinical case report presents the clinical sequence of the surgical treatment of a supernumerary tooth in transverse position. The surgical treatment (extraction) of the supernumerary teeth are indicated in parts included and which are also within the dental arches; This Odontogenic pathology tends to be found sporadically during the examinations; however, not dealing with it and deal with it can become another dental pathology involving parts adjacent to the lesion. Presents the clinical sequence of the surgical approach to extract a supernumerary tooth in a transverse position.

Keywords: Supernumerary tooth, extractions, pediatric dentistry.

\section{Víctor Velásquez Reyes ${ }^{1}$, Yuri Castro Rodriguez ${ }^{2}$}

\author{
1 Docente del Departamento Académico de Estoma- \\ tología Pediátrica FO UNMSM \\ 2 Estudiante de la Facultad de Odontología, Univer- \\ sidad Nacional Mayor de San Marcos
}

Correspondencia:

Víctor Manuel Velásquez Reyes

Facultad de Odontología,UNMSM.Av. Germán Amézaga s/n , Lima 1. Perú

Teléfono : 999177282

Correo electrónico: v.velasquez.r@gmail.com

Fecha de recepción: 21-11-2013

Fecha de aceptación: 28-11-2013

\section{Introducción}

Los dientes son parte fundamental en el sistema estomatognático ya sea en los niños como en los adultos. Las enfermedades bucales de mayor prevalencia aún continúan siendo la caries y la enfermedad periodontal ${ }^{1}$; sin embargo, existen otras alteraciones bucales de menor prevalencia que deben ser consideradas al momento de analizar y diagnosticar a un determinado paciente. Dentro de los otros tipos de patología bucal se encuentran las alteraciones del número dentario ${ }^{2}$; dentro de esta clasificación se encuentran las anodoncias (ya sean las anodoncias totales y parciales $)^{3}$ y los dientes supernumerarios ${ }^{4}$. Los dientes supernumerarios son definidos como dientes en exceso sobre el número nor$\mathrm{mal}^{5}$ Aunque estos dientes pueden presentarse en cualquier localización, tiene predilección por ciertas localizaciones ${ }^{6}$ Son mucho más frecuentes en el maxilar $(90 \%)^{7,8}$ que en la mandíbula (10 $\%)^{9,10}$ El más frecuente es un diente supernumerario localizado entre los incisivos centrales superiores que suele designarse como mesiodens ${ }^{11}$ seguido por los cuartos molares superiores y los incisivos laterales superiores ${ }^{11,12} \mathrm{Un}$ diente supernumerario puede parecerse al diente normal correspondiente o puede tener una conformación rudimentaria y cónica, con un parecido escaso o nulo a su homólogo normal ${ }^{13} \mathrm{El}$ diagnóstico de una pieza supernumeraria muchas veces es difícil de identificar en un examen de rutina, su presencia se ve evidenciada a través de exámenes auxiliares siendo la radiografía panorámica la que principalmente nos indica su presencia $^{14}$. El manejo adecuado en la clasificación, diagnóstico y tratamiento debe ser parte de un abordaje multidisciplinario para evitar problemas clínicos asociados con su etiología durante la práctica odontológica ${ }^{15}$ muchas veces serán necesarias exámenes específicos para localizar al diente supernumerario, la técnica horizontal para la toma radiográfica y los distintos tipos de tomografías son útiles para obtener la ubicación precisa del diente supernumerario ${ }^{16}$.

El tratamiento depende sobre el tipo y posición de los supernumerarios y sus efectos sobre la dentición adyacente. El plan de tratamiento debe estar basado en un tratamiento integral, considerando la edad y tipo de mal oclusión. La exploración clínica debe realizarse en cada caso, en la cual se deben contar los dientes por cuadrante, verificar la secuencia de erupción dentaria y si existen signos anormales de erupción ${ }^{17}$ La exodoncia de un supernumerario está indicada cuando: hay retardo de la erupción de los incisivos permanentes, por desplazamiento de los incisivos, pacientes de labio y paladar hendido en donde se les va hacer un injerto óseo, cuando hay que realizar un implante, en presencia de signos de alargamiento del folículo, supernumerarios ya erupcionados $^{18} \mathrm{El}$ presente reporte de caso clínico tiene como objetivo mostrar la secuencia clínica del abordaje quirúrgico para extraer una pieza supernumeraria incluida.

\section{Descripción del caso clínico}

Se reporta el caso clínico de un niña de 12 años, sin enfermedades sistémicas ni otras patologías, que acude a consulta a la clínica de odontología de la facultad de odontología de la Universidad Nacional Mayor de San Marcos para realizársele la corrección de las mal posiciones dentarias pues, al examen clínico se observan mal posiciones dentarias 
superiores e inferiores, desviación de la línea media dentaria inferior, ausencia de las piezas 15, 36 y 45así como la persistencia de la pieza 65 (Fig. 1 y 2).

Al examen radiográfico se confirma la ausencia de la pieza 36, la retención de la pieza 45 y la presencia de una imagen radiopaca a nivel apical de la pieza 65 compatible con una lesión odontogénica de tipo tumor. Al examen periapical se visualiza que dicha lesión es compatible con una pieza supernumeraria incluida. A través de la toma radiográfica con la técnica horizontal se confirma la posición transversal de dicha pieza supernumeraria. (Figuras 3 y 4 ).

\section{Diagnóstico}

Se diagnostica alteración en el número de las piezas dentarias con la presencia de una pieza supernumeraria a nivel apical de la pieza 65 así como la per-

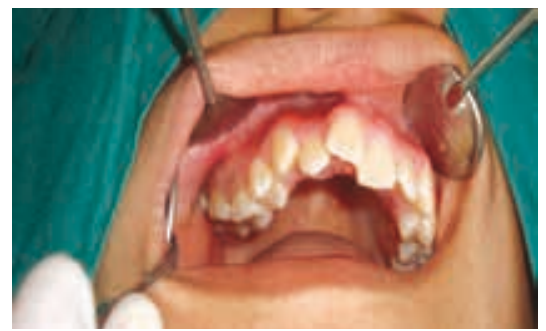

Fig. 1 Alteración Oclusal

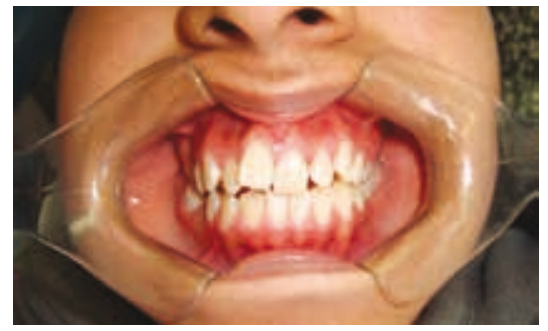

Fig. 2 Desviación de la línea media

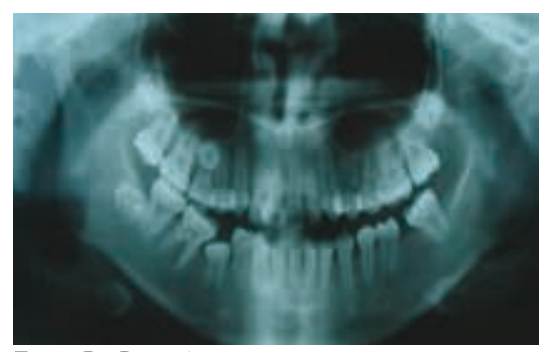

Fig. 3 Rx Panorámica

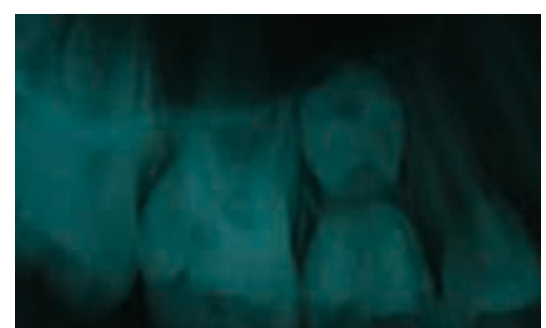

Fig. 4 Rx Periapical sistencia de la pieza 65 dentro de la arcada. Así mismo se diagnóstica enfermedad gingival leve asociada a placa bacteriana, lesiones cariosas incipientes en piezas permanentes y mal posición dentaria con apińamiento dentario superior e inferior.

\section{Plan de tratamiento}

Se planifica un tratamiento integral que abarca tratamiento periodontal, obturación de fosas y fisuras de forma preventiva, exodoncia del diente supernumerario y corrección de la mal posición dentaria.

\section{Tratamiento quirúrgico}

El tratamiento quirúrgico de la pieza supernumeraria consistió en la extracción de la misma. La intervención quirúrgica

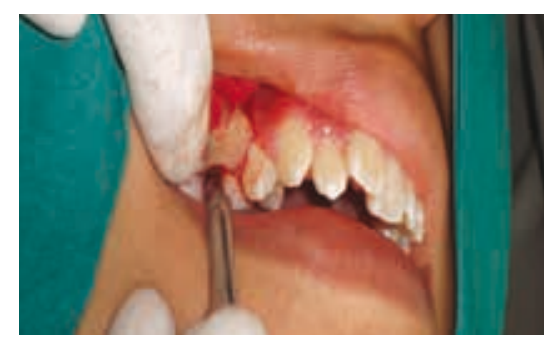

Fig. 6 Incisión

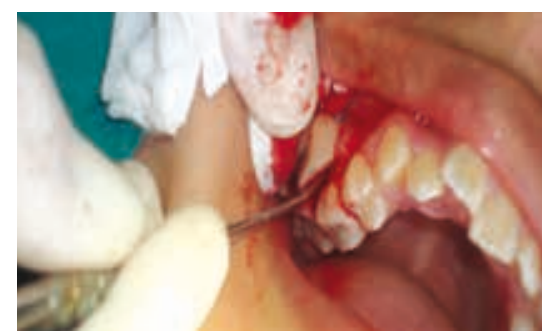

Fig. 7 Elevación de colgajo

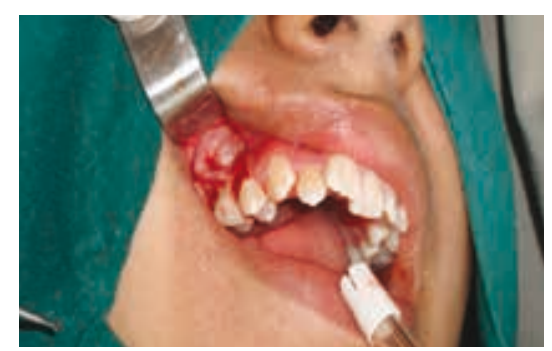

Fig. 8 Osteotomía

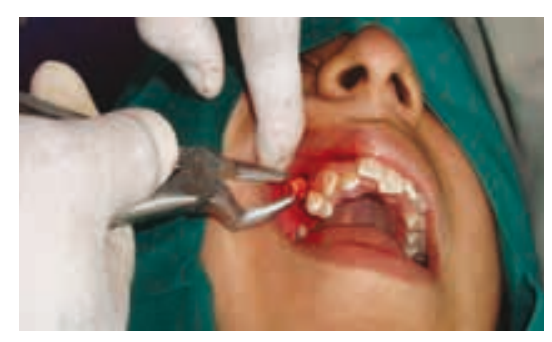

Fig. 9 Exodoncia del supernumerario se realizó con anestesia local (lidocaína al $2 \%$ con epinefrina 1: 80000), el primer paso fue la realización de incisiones a espesor total y a deflexión total (Fig. 6), luego se elevó un colgajo a espesor total a partir de las incisiones (Fig. 7), una vez obtenido visibilidad de la zona ósea a trabajar se procedió a la osteotomía del mismo hasta tener acceso a la pieza supernumeraria (Fig. 8).

Una vez logrado el acceso a la pieza supernumeraria esta se extrajo a través del uso del botador y posteriormente a través del uso del fórceps (Fig. 9), también se extrajo la pieza decidua persistente para facilitar el acceso a la pieza supernumeraria (Fig. 10), una vez extraída la pieza se procedió a tratar la cavidad dejada por la pieza supernumeraria, y a la regularización de las espículas óseas y al lavado de la cavidad (Figura 11).

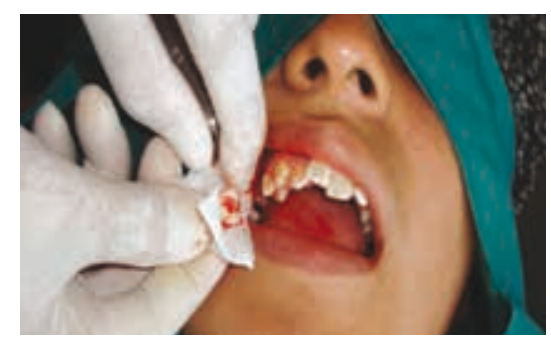

Fig. 10 Supernumerario

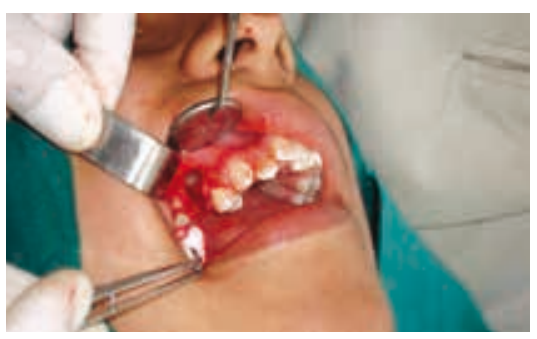

Fig. 11 Lecho vacío

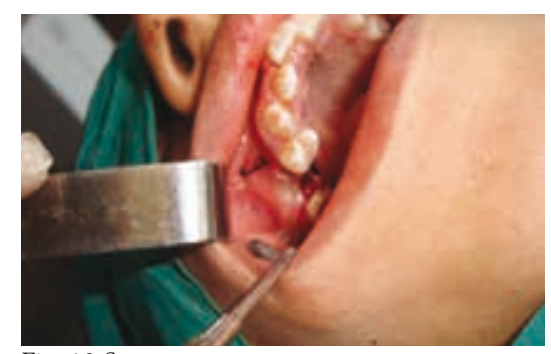

Fig. 12 Sutura

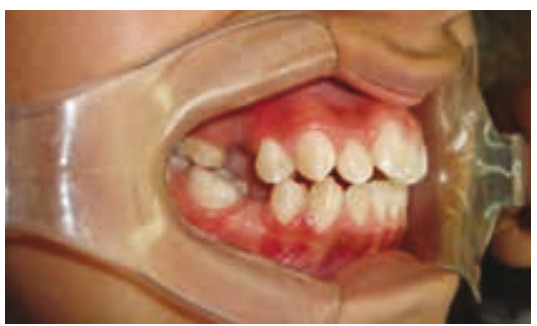

Fig. 13 Cicatrización

Odontol. Sanmarquina 2013; 16(2):47-49 
Al final se suturó el colgajo levantado, evitando que en algún punto se liberen las incisiones y se facilite la integridad celular (Fig. 12). El control a las dos semanas pos tratamiento quirúrgico mostró una completa resolución de la inflamación y cicatrización de los tejidos blandos (Fig. 13).

\section{Discusión}

La ausencia clínica de dientes permanentes que hayan sobrepasado su tiempo estimado de erupción nos hace suponer de la presencia de problemas que pudiesen contribuir a la alteración en dicho proceso eruptivo, como por ejemplo: inclusiones dentarias (dientes impactados en hueso), presencia de diente supernumerarios, odontomas, quistes dentígeros, entre otros.

La presencia de dientes supernumerarios, caninos en transposición, y odontomas representan un serio problema y necesariamente deben ser tratados multidisciplinariamente ${ }^{4}$ En nuestro caso la persistencia de la pieza 65 y la ausencia de la pieza 25 nos hicieron pensar en la existencia de alguna alteración intraósea en dicho sector.

Los dientes supernumerarios suelen ser problemas poco comunes dentro de la patología bucal, su prevalencia suele oscilar entre el 4 a $10 \%{ }^{10}$ La radiografía panorámica, las radiografías de localización, y la tomografía lineal fueron muy útiles para complementar el examen clínico y contribuir al diagnóstico del paciente ya que los problemas que se presentaron asociadas a los dientes incluidos fueron hallazgos radiográficos y difíciles de localizar. Sin embargo, no debemos confiarnos totalmente en el informe radiográfico, debemos usarlo como una guía de inicio, revisarlo varias veces y tratar de tener una mayor cantidad de estudios con el uso de las diferentes técnicas radiográficas conocidas y de esta forma obtener datos más precisos sobre la localización de estos problemas, pues muchas veces como en nuestro caso, pueden presentarse como casos límite en la interpretación radiográfica $^{1}$ En nuestro caso el diagnóstico de la pieza supernumeraria se logró a través de la radiografía panorámica y localizada a través de las radiografías periapicales con la técnica horizontal.

\section{Conclusiones}

Es importante en un paciente niño, que se encuentra en pleno proceso de crecimiento y desarrollo, la correlación del examen clínico con la edad cronológica en la evaluación de la cavidad bucal, teniendo en cuenta la presencia o no de los dientes respectivos tanto en las arcadas superiores como inferiores.

Recurrir a las diferentes técnicas radiográficas, ya que como en éste caso, la interposición de imágenes es un obstáculo para realizar una buena evaluación.

Se optó por la alternativa quirúrgica y la paciente fue evaluada obteniendo resultados favorables.

\section{Referencias bibliográficas}

1. Olate S, Alister JP, Muñante JL, Pozzer L, Albergaria-Barbosa JR. Tratamiento Quirúrgico de Caninos Mandibulares Bilaterales Transmigrados. Int J Odontostomat. 2010;4(3):285-90.

2. Prado J, Perea M. Múltiples dientes supernumerarios y odontoma en dentición permanente: Reporte de caso. Odontol Pediatr. 2008;7(1):29-33.

3. Canaval C, Perea M, Kanashiro C, Mayo A. Dientes supernumerarios en odontopediatría. Rev Estomatol Herediana. 2003;13(1-2):27-9.

4. Chu FC, Li TK, Lui VK, Newsome PR, Chow RL, Cheung LK. Prevalence of impacted teeth and associated pathologies--a radiographic study of the Hong Kong Chinese population. Hong Kong Med J. 2003;9(3):158-63.

5. Sivapathasundharam B, Einstein A. Non-syndromic multiple supernumerary teeth: report of a case with 14 supplemental teeth. Indian J Dent Res. 2007;18(3):144.

6. Boj J. Desarrollo y erupción dentaria en Odontopediatría. Barcelona, España: Editorial Masson S.A; 2005.

7. Sasaki H, Funao J, Morinaga H, Nakano K, Ooshima T. Multiple supernumerary teeth in the maxillary canine and mandibular premolar regions: a case in the postpermanent dentition. Int J Paediatr Dent. 2007;17(4):304-8.
8. Nuñez-Martínez JM, Sierra J, García-López S, Tenorio-Torres G, Osorno C. Distribución y localización de los dientes supernumerarios en nińos y adultos en una población mexicana: estudio radiográfico retrospectivo. Rev Cien Clín. 2007; 8(2):59-63.

9. Solares R, Romero MI. Supernumerary premolars: a literatura review. Pediatr Dent. 2004;26(5): 450-8.

10. Ashkenazi M, Greenberg BP, Chodik G, Rakocz M. Postoperative prognosis of unerupted teeth alters removal of supernumerary teeth or odontomas. Am J Orthod Dentofacial Orthop. 2007; 131(5):614-9.

11. Wang XX, Zhang J, Wei FC. Autosomal dominant inherence of multiple supernumerary teeth. Int J Oral Maxillofac Surg. 2007; 36(8):756-8.

12. Acikgoz A, Acikgoz G, Tunga U, Otan F. Characteristics and prevalence of non-syndrome multiple supernumerary teeth: a retrospective study. Dentomaxillofac Radiol. 2006; 35(3):185-90.

13. Kim S-G, Lee S-H. Mesiodens. A clinical and radiographic study. J Dent Child 2003; 70(1):58-60.

14. Di Santa J, Betancour O. Tratamiento Ortodontico-Quirurgico de los Dientes Supernumerarios: Presentación De un Caso Clínico. Acta. Odontológica de Venezuela. 2008;48(1):1-8.

15. De Oliveira C, Neves S, Correia B, Neves E, Alves R. A survey of 460 supernumerary teeth in Brazilian children and adolescents .Int J Paeditric Dent. 2008; 18(2):98-106.

16. Kangowska-Adamczÿk H, Karmariska B. Similar locations of impacted and supernumerary teeth in monozygotic twin: A report of 2 cases. Am J Orthod Dentofac Orthop. 2001; 119(1):67-70.

17. Laskaris G. Patologías de la cavidad bucal en niños y adolescentes. Colombia. AMOLCA; 2001.

18. Soldevilla L, Oriel O, Rodríguez E. Desinclusión de dientes retenidos mediante aparatología removible. Odontología Sanmarquina.2003;6(12):41-5. 